\title{
Self-consistent study of electron confinement to metallic thin films on solid surfaces
}

\author{
E. Ogando, ${ }^{1, *}$ N. Zabala, ${ }^{1,2}$ E. V. Chulkov, ${ }^{2,3}$ and M. J. Puska ${ }^{4}$ \\ ${ }^{1}$ Elektrika eta Elektronika Saila, Zientzia eta Teknologia Fakultatea UPV-EHU 644 P.K., 48080 Bilbao, Spain \\ ${ }^{2}$ Donostia International Physics Center (DIPC) and Centro Mixto CSIC-UPV/EHU, 20080 Donostia, Spain \\ ${ }^{3}$ Materialen Fisika Saila, Kimika Fakultatea, UPV-EHU 1072 P.K., 20080 Donostia, Spain \\ ${ }^{4}$ Laboratory of Physics, Helsinki University of Technology, P.O. Box 1100, FIN-02015 HUT, Finland
}

(Received 6 July 2004; revised manuscript received 22 November 2004; published 10 May 2005)

\begin{abstract}
We present a method for density-functional modeling of metallic overlayers grown on a support. It offers a useful tool to study nanostructures, combining the power of self-consistent pseudopotential calculations with the simplicity of a one-dimensional approach. The model is illustrated for $\mathrm{Pb}$ layers grown on the $\mathrm{Cu}(111)$ surface. The analysis provides the strength of the electron confinement barriers in thin slabs with accuracy, supporting the interpretation of the quantum well state spectra measured by scanning tunneling spectroscopy. On the other hand, it offers a benchmark to check the simple analytical models commonly used in the literature to study metallic films on semiconducting or metallic surfaces. As a result, some deficiencies are detected in the applicability of those models, which often lead to an overestimation of the number of wetting layers. Finally, an improved formula is proposed.
\end{abstract}

DOI: 10.1103/PhysRevB.71.205401

PACS number(s): 68.35.-p, 73.21.Fg, 71.15.Mb

\section{INTRODUCTION}

The ability to manufacture nanostructures on solid surfaces by controlled-growth or atomic-manipulation techniques has increased enormously during the last decades. ${ }^{1,2}$ At the same time, spectroscopic methods to study different physical properties and phenomena of these structures have also experienced huge development. In understanding and interpreting the ensuing experimental results, rich in quantum phenomena, accurate theoretical and computational modeling plays a vital role.

A widely studied phenomenon is the growth of thin $\mathrm{Pb}$ films or extended $\mathrm{Pb}$ islands on solids, for example, on $\mathrm{Si}$ or $\mathrm{Cu}$ surfaces. These systems provide a laboratory to test the so-called quantum size effects (QSE) arising because of the electron confinement perpendicular to the surface. The confinement results in discrete energy levels, the so-called quantum well states (QWSs). With the increasing film thickness, QWSs become occupied, producing oscillations in the total energy, work function, and other physical properties. The oscillations in energy are the origin of the "magic" islands heights. ${ }^{3-16}$ In a previous publication ${ }^{13}$ we successfully applied a one-dimensional (1D) pseudopotential model to gain physical insight into the magic heights of $\mathrm{Pb}$ islands on $\mathrm{Cu}(111)$, by studying the energetics of that system. In fact, the beating pattern obtained has been measured in the $\mathrm{Pb} / \mathrm{Si}(111)$ system. ${ }^{17}$ In contrast, in the present paper we focus on the determination of confinement barriers.

The most important feature characterizing an overlayer or a nanoisland is its height. However, measurements based on different physical processes may provide different values. $\mathrm{X}$-ray diffraction ${ }^{18}$ can be used to directly determine the number of atomic monolayers (ML) in thin films. Scanning tunneling microscopy $(\mathrm{STM})^{3}$ gives the thickness of finite nanoislands. In helium atom scattering (HAS) the specular returning point of He atoms is measured. ${ }^{4,19}$ STM and HAS reflect the electron-density profile of the surface., ${ }^{4,19}$ Scan- ning tunneling spectroscopy (STS) measures the QWS spectrum, and the width of the quantum well confining the electrons is evaluated from it. ${ }^{20}$ In addition to the overlayervacuum surface profile, the QWS spectrum is also sensitive to properties of the substrate-overlayer interface. The information about the confining quantum well is important not only for determing the film thickness but also for the understanding and controlling the so-called electronic growth mode.

$\mathrm{Pb}$ islands grown on $\mathrm{Cu}(111)$ were recently studied by Otero et al. ${ }^{20}$ They fitted the QWS spectra measured by STS using the finite-potential-well model and unexpectedly found that, actually, the infinitely high-potential barriers give a better fit than the more realistic finite barriers. There are also recent STS measurements of QWSs in $\mathrm{Pb}$ layers on $\mathrm{Si}(111){ }^{11,21,22}$ The effective thickness of the $\mathrm{Pb}$ overlayer is then obtained by fitting the energy differences of the consecutive states. This is not very accurate and, in fact, there are contradictory results in the literature on the thickness of the wetting layer, ranging from 1 ML (Refs. 7-9) to 3 ML. ${ }^{10,11,21,22}$ Our aim is to develop the formalism for the determination of confinement barriers in order to resolve the dilemma of these measurements of $\mathrm{Pb}$ on $\mathrm{Cu}$ or $\mathrm{Si}$ substrates.

There are several theoretical works devoted to the study of QSE in thin films, within the density functional theory (DFT). First-principles atomistic approaches ${ }^{23-31}$ or jellium models ${ }^{32-36}$ have been used. Usually, because of the high computational demand, the substrate is not included, and the electronic structure is calculated for a slab describing the overlayer only. The work by Hong et al. ${ }^{37}$ for $\mathrm{Pb} / \mathrm{Si}(111)$ is one exception. On the other hand, there are several simple analytical models used for the confinement barriers, ${ }^{20,21,38}$ but a priori assumptions about the barrier type, its position, or quantum numbers of the measured states can lead to an erroneous interpretation of the experiments. The simplifications made in the modeling effectively hinder the analysis of the experimental results. 
In the present work we perform self-consistent electronic structure calculations, including the effect of the substratefilm boundary, so that the penetration of the QWS into the substrate is realistically described. Second, with our selfconsistent results we study the above-mentioned simple analytical models and point out their deficiencies as well as the most important factors for the proper description of the electron confinement. This knowledge is especially important when using these models in analyzing the STS results for completely covered substrates or for systems with wetting layers of unknown thicknesses. A pragmatic aim of the present paper is to document the construction of the unscreened 1D pseudopotential and provide a simple parametrization that can be used in future studies, e.g., for different nanostructures on surfaces.

In Sec. II we report the construction of the $\mathrm{Cu}(111)$ pseudopotential and the resulting self-consistent electronic structures for the $\mathrm{Pb} / \mathrm{Cu}(111)$ system. In Secs. III and IV we describe analytical models to calculate the confinement barriers, whose reliability is analyzed by applying them to the results of the self-consistent calculations. Finally, in Sec. V an improvement to the analytical expressions is introduced in order to acquire accuracy in their determination. Section VI contains the conclusions of the work. Atomic units (i.e., $\hbar$ $=e^{2}=m=1$ and distances measured in Bohr radius units $a_{0}$ $=0.53 \AA$ ) will be used throughout this work, unless otherwise specified.

\section{ONE-DIMENSIONAL PSEUDOPOTENTIAL MODEL}

\section{A. Construction of the pseudopotential}

The present calculations are performed in the framework of the DFT (Ref. 39) within the local density approximation (LDA). ${ }^{40,41}$ Instead of finite $\mathrm{Pb}$ islands we consider infinitely extended films on the $\mathrm{Cu}$ surface. This is justified because in the experiments ${ }^{3,20}$ considered the characteristic lateral dimension of the $\mathrm{Pb}$ islands is around $1000 a_{0}$, so that the lateral electron-confinement effects are irrelevant. We assume perfect translational invariance, i.e., a homogeneous freeelectron gas, along the surface ( $x y$ plane). Hence, singleparticle wave functions are taken to be of the form

$$
\Phi(\mathbf{r})=\psi_{n}(z) e^{i \mathbf{k}_{\|} \cdot \mathbf{r}_{\|}},
$$

where $\psi_{n}(z)$ is the wave function in the direction perpendicular to the surface, and plane waves are used for the surface parallel directions. The eigenenergies are given by

$$
\varepsilon_{n k_{\|}}=\varepsilon_{n}+\frac{k_{\|}^{2}}{2}
$$

where $\varepsilon_{n}$, being the eigenvalue of the $n$th perpendicular state $\psi_{n}(z)$ describes the bottom of the $n$th subband. Kohn-Sham equations are solved numerically only in the $z$ direction (1D problem) that enables the calculation of electron wave functions extending deep into the $\mathrm{Cu}$ substrate and the modeling of systems having tens of $\mathrm{Pb}$ ML. The real-space MIKA package $^{42-44}$ for electronic structure calculations has been used to solve these equations within a model of finite and periodic systems in the $z$ direction with Dirichlet and periodic boundary conditions used, respectively.
The effective or screened potential of the Kohn-Sham ${ }^{39}$ equations in the $z$ direction is written as

$$
V_{\mathrm{eff}}(z)=\int \frac{n_{-}\left(z^{\prime}\right)-n_{+}\left(z^{\prime}\right)}{\left|r-r^{\prime}\right|} d r^{\prime}+V_{\mathrm{xc}}\left[n_{-}(z)\right]+V_{p s}(z),
$$

where the first term on the right-hand side is the Hartree term $V_{H}(z)$, which includes the electron density $n_{-}(z)$ and the neutralizing rigid positive charge density $n_{+}(z)$. The second term gives the LDA exchange-correlation potential. The third term accounts for the pseudopotential that improves the simple jellium scheme. For the supported overlayer system, $V_{p s}(z)$ has two contributions, one from the $\mathrm{Pb}$ overlayer and the other from the $\mathrm{Cu}(111)$ substrate. The free electronlike character of $\mathrm{Pb}$ at the Fermi level justifies the use of the stabilized jellium or averaged pseudopotential ${ }^{27,45,46}$ approach to model $\mathrm{Pb}$. In practice, the jellium model allows us to simulate any $\mathrm{Pb}$ overlayer thickness. ${ }^{13}$ The $\mathrm{Pb}$ contribution to $V_{p s}(z)$ stabilizing the electron gas at the density ( $n$ $=3 / 4 \pi r_{s}^{3}$ ) corresponding to $r_{s}=2.30 a_{0}$ is a constant shift $V_{\text {stab }}$ relative to the vacuum level restricted to the region of the positive background charge. The stabilized $\mathrm{Pb}$ provides a proper work function so that the spilling of the electron density into the vacuum is well described. It also gives a proper value for the bottom of the valence electron band. This guarantees the correct Fermi wavelength $\lambda_{F}$, which is of crucial importance for the properties related to the electron confinement.

For the $\mathrm{Cu}(111)$ support we have obtained a 1D pseudopotential (see Appendix A), with the positive background density of $r_{s}=2.55 a_{0}$ and a surface profile given by a Fermilike distribution

$$
n_{+}(z)=\frac{n_{+}(\mathrm{Cu})}{e^{\left(z-D_{0}\right) / \Delta z}+1},
$$

where $D_{0}$ is the surface edge position and $\Delta z=0.09 a_{0}$ accounts for the smoothing (for numerical reasons) at the edge.

The 1D pseudopotential obtained in Appendix A correctly reproduces the experimental $\mathrm{Cu}(111)$ work function and the [111]-projected band structure, including the band gap. In this way, we also obtain the correct confinement potential at the $\mathrm{Cu}(111)-\mathrm{Pb}$ interface. The method presented for its generation is extensible to other substrates as well and, for the system considered, it can be fitted using the form of Eq. (A1) with $A_{10}=-1.89 \mathrm{eV}$ and $A_{1}=5.01 \mathrm{eV}$.

\section{B. Electronic structure}

The main results of this paper are obtained with the pseudopotential for the $\mathrm{Cu}(111)$ surface and the stabilized jellium model for the $\mathrm{Pb}$ overlayer. In the inset of Fig. 1 we sketch the geometry used in the calculations. In the middle there are $25 \mathrm{ML}$ of $\mathrm{Cu}$ and $\mathrm{Pb}$ overlayers corresponding up to $23 \mathrm{ML}$ (one $\mathrm{ML}$ of $\mathrm{Pb}$ is $5.4 a_{0}$ ) are attached symmetrically on both sides. Complementary calculations have been performed for unsupported $\mathrm{Pb}$ slabs as well.

Figure 1 shows the electron and positive background densities corresponding to the coverage of $4 \mathrm{ML}$ of $\mathrm{Pb}$. We note six Friedel oscillations in the Pb electron density, with the 


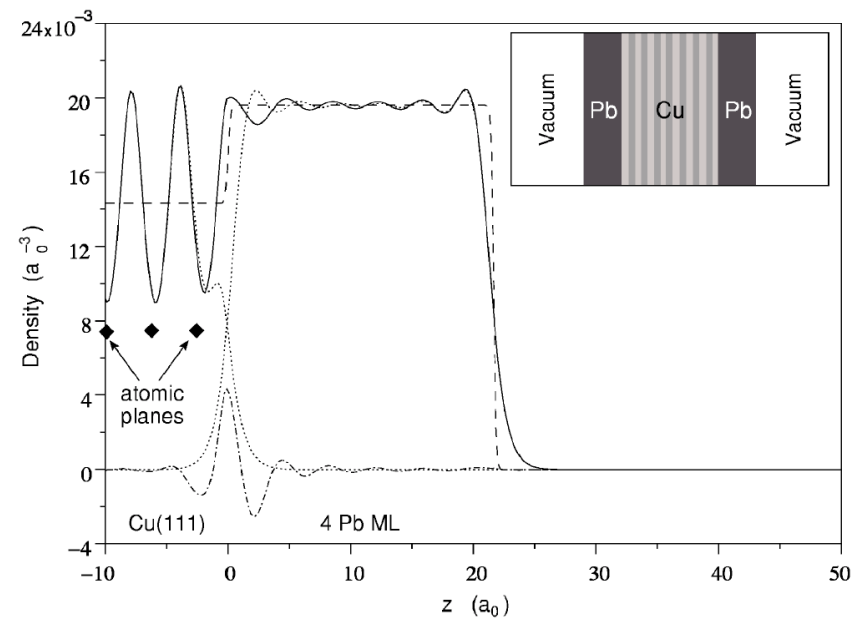

FIG. 1. Electron density (solid line) and average positive background density (dashed line) for $4 \mathrm{ML}$ of $\mathrm{Pb}$ on $\mathrm{Cu}(111)$. The origin $z=0$ is at the $\mathrm{Cu}-\mathrm{Pb}$ interface. The dotted curves show the electron densities of the free-standing $\mathrm{Cu}(111)$ and $\mathrm{Pb}$ slabs. The dasheddotted curve shows the charge transfer in the combined system relative to the free-standing $\mathrm{Cu}(111)$ and $\mathrm{Pb}$ slabs. The inset sketches the symmetric geometry used in the calculations in the $z$ direction.

wavelength of about half the Fermi wavelength. This kind of well-developed Friedel oscillation pattern commensurate with the thickness of the overlayer is characteristic for the stable "closed shell" or "magic" overlayer systems. In $\mathrm{Cu}(111)$ the electron density oscillates strongly, according to Fig. 1, as a consequence of the oscillating pseudopotential. The electron densities of the corresponding free-standing $\mathrm{Cu}(111)$ and $\mathrm{Pb}$ slabs are also given for reference. It is remarkable that the electron density moves from $\mathrm{Pb}$ toward $\mathrm{Cu}(111)$, indicating a possible increase in the effective width of the $\mathrm{Pb}$ slab. The charge transfer obtained when comparing the $\mathrm{Pb} / \mathrm{Cu}(111)$ system with its free-standing counterparts $\left(\Delta n_{-}=n_{-}[\mathrm{Pb} / \mathrm{Cu}(111)]-n_{-}[\mathrm{Cu}(111)]-n_{-}[\mathrm{Pb}]\right) \quad$ reveals charge accumulation at the interface. A small amount of charge ( $\sim 5 \%$ of one $\mathrm{Pb}$ ML charge) is transferred from $\mathrm{Pb}$ to $\mathrm{Cu}$ in order to equilibrate their chemical potentials, $-4.1 \mathrm{eV}$ and $-4.94 \mathrm{eV}$ for $\mathrm{Pb}$ and $\mathrm{Cu}$, respectively.

In Fig. 2(a) the effective potential $V_{\text {eff }}$ and its components are shown; the total pseudopotential $V_{p s}$ is not plotted for clearness. The dark gray shadowing gives the rough spatial extension where the localized QWSs can appear because of the $\mathrm{Cu}(111)$ energy gap. The light gray area marks the potential well between the vacuum barrier and the $\mathrm{Pb}-\mathrm{Cu}$ interface barrier. This well is created because the potential in $\mathrm{Pb}$ is $\sim 2 \mathrm{eV}$ lower than the average $\mathrm{Cu}$ potential (note the dashdotted curve corresponding to the stabilized jellium calculation $\left.V_{S J}\right)$.

In Fig. 2(b) the eigenvalues are plotted as a function of the wave vector $k_{z}$. The QWSs fall on the dotted parabola, which is the free-electron-model band for $\mathrm{Pb}$. The states extending over the whole $\mathrm{Cu}-\mathrm{Pb}$ system and forming a continuous band are plotted with open circles. The nearly parabolic band reflects the free-electron character in our description of the $\mathrm{Cu}$ substrate. The QWSs in the lower, light gray area have a minor relevance on the electronic properties of the system. In contrast, QWSs in the upper dark gray area play an important role because increasing the $\mathrm{Pb}$ thickness lowers the QWS energy and they become occupied one by one, producing the oscillations in the electronic properties.

\section{INTERPRETATION OF STS EXPERIMENTS}

Scanning tunneling spectroscopy is capable of resolving energy levels of QWSs. Nevertheless, the interpretation of the experimental results is still difficult. ${ }^{7,20}$ Our model pro-
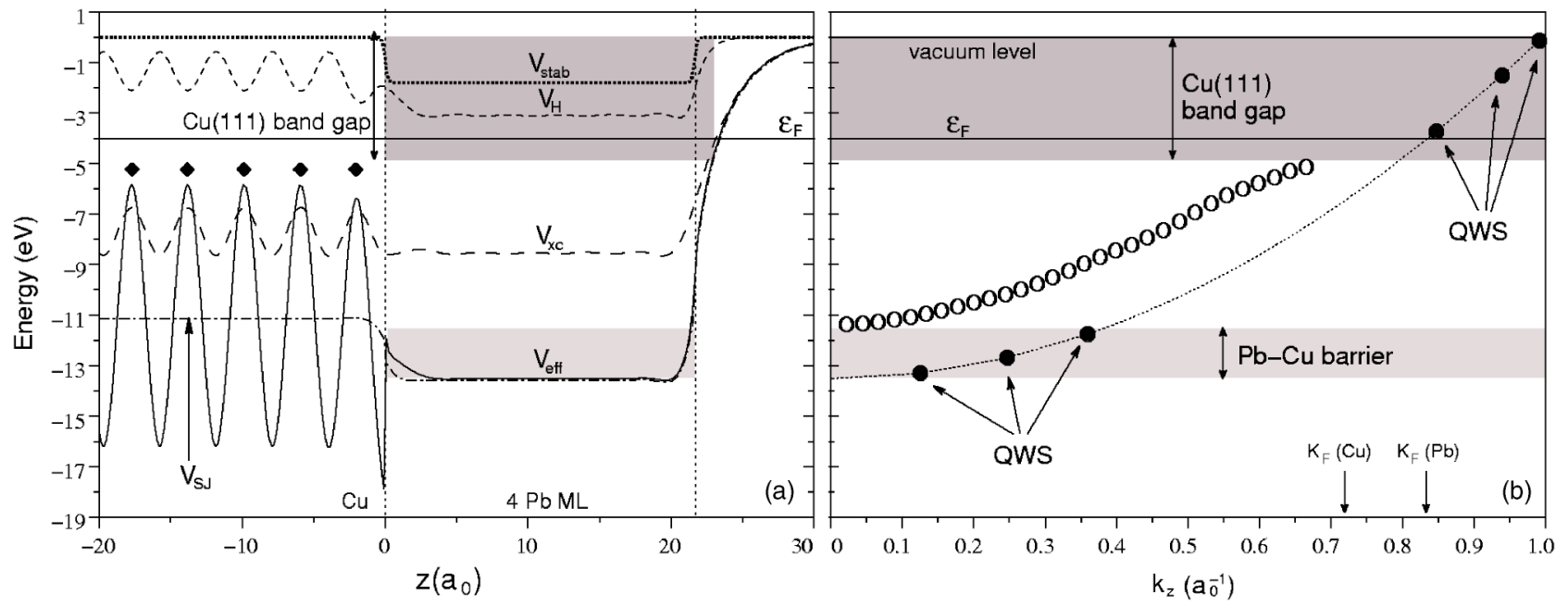

FIG. 2. (a) Effective potential $V_{\text {eff }}(z)$ (solid line) and its components for $4 \mathrm{ML}$ of $\mathrm{Pb}$ on $\mathrm{Cu}(111)$. The origin $z=0$ is at the $\mathrm{Cu}-\mathrm{Pb}$ interface and the energies are measured with respect to the vacuum level. The potential obtained with the stabilized jellium Cu is shown as well (dashed-dotted line). The vertical dotted lines represent the edges of the Pb slab, and the diamonds mark the atomic Cu planes. (b) Energy eigenvalues as a function of the wave vector $k_{z}$. States extended over the whole system (open circles) and QWSs trapped in the Pb layer (filled circles) are distinguished. The dark and light gray areas correspond to the $\mathrm{Cu}(111)$ energy gap and the potential well in $\mathrm{Pb}$, respectively. The free-electron-model band parabola in $\mathrm{Pb}$ (dotted line) and the $\mathrm{Cu}$ and $\mathrm{Pb}$ Fermi wave vectors are marked for reference. 


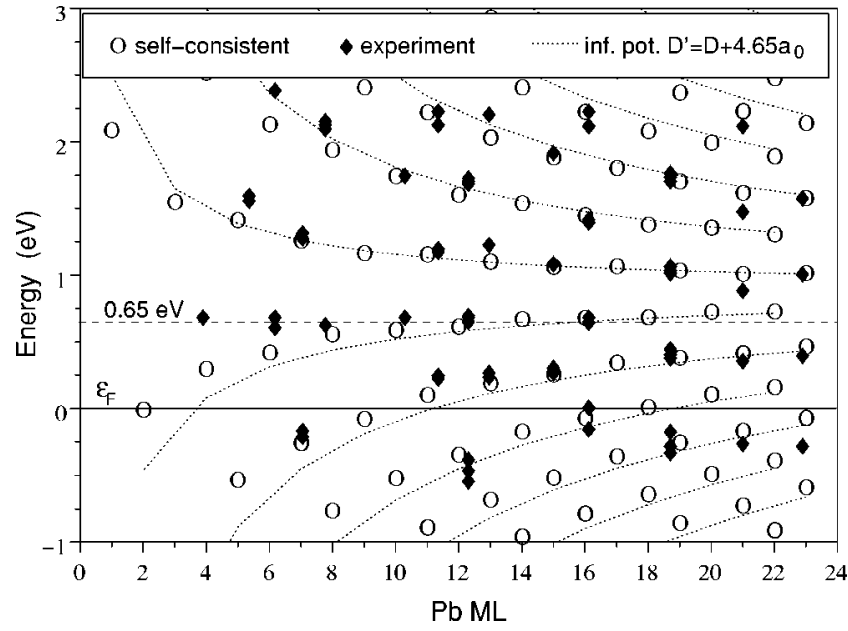

FIG. 3. Energy eigenvalues of QWSs in the Pb overlayer on the $\mathrm{Cu}(111)$ surface as a function of the number $\mathrm{Pb} \mathrm{ML}$. Open circles and filled diamonds give the calculated and experimental STS values, ${ }^{20}$ respectively. The dotted curves link points calculated with the infinite-potential-well model (see Appendix B) with the effective well width of $D^{\prime}=D+4.65 a_{0}$.

vides a good tool to enlighten the problems encountered and to suggest a simple but realistic picture with relevant parameters.

Figure 3 shows the calculated eigenvalues as a function of the number of $\mathrm{Pb}$ ML. The comparison with the QWS energy levels measured by Otero et al. ${ }^{20}$ shows a good agreement for large coverage heights. Below $6 \mathrm{ML}$ the correspondence is a bit worse. This can be because of the absence of real crystal structure of the $\mathrm{Cu}-\mathrm{Pb}$ interface in our model as well as to the interaction with the $\mathrm{Cu} d$ electrons omitted in the calculations. The importance of these phenomena decreases as the number of $\mathrm{Pb} \mathrm{ML}$ increases.

To explain the measured energy eigenvalues Otero et al. ${ }^{20}$ tried the finite-square-potential-well model first but the fit was not satisfactory. However, they obtained a very good agreement by using the infinite-square-potential-well model or by decreasing the width of the finite-square well by $\sim 2.6 a_{0}$.

The fact that the infinite-potential well produces much better results than the more realistic finite-potential well is counterintuitive. It has been proven for slabs, ${ }^{32,47}$ wires $^{48,49}$ and clusters ${ }^{50}$ that the real potential profiles are soft. In fact, it can be seen in Fig. 2(a) that the self-consistent potential is soft, with an effective width increasing as a function of the QWS energy level. On the other hand, this effect seems not to be counterbalanced by the confining potential on the $\mathrm{Cu}-\mathrm{Pb}$ interface. The QWSs penetrate inside the $\mathrm{Cu}(111)$ (see Fig. 5) and the electron density in Fig. 1 moves toward $\mathrm{Cu}$. Nevertheless, a very good agreement with the experiments is obtained with both the infinite-square-potential-well model and soft self-consistent potentials. In addition, a good fit is also obtained with the infinite-square-potential well, but by using increased well widths in order to take into account the wave-function spill out (dotted lines in Fig. 3).

\section{ANALYSIS WITHIN THE PHASE-ACCUMULATION MODEL}

\section{A. Phase shifts}

In order to clarify the reasons of contradictions or dissimilar results mentioned above, here we use the phaseaccumulation model (see Appendix B) to evaluate the phase shifts for the confinement barriers in our self-consistent calculations. We use the unsupported $\mathrm{Pb}$ slab to calculate the phase shift $\phi_{\mathrm{Pb} \text {-vac }}$ and then we evaluate the phase shift $\phi_{\mathrm{Cu}(111)-\mathrm{Pb}}$ in the $\mathrm{Pb} / \mathrm{Cu}(111)$ system. The procedure used is to choose a QWS with an energy just below the vacuum level and then to identify the corresponding quantum number $n$. After the wave vector $k_{z}$ is calculated from the kinetic energy $\varepsilon=k_{z}^{2} / 2$, the phase shift $\phi_{\mathrm{Pb} \text {-vac }}$ is evaluated for the QWS energy level by Eq. (B1) for the unsupported Pb slab. Increasing the slab thickness, the energy eigenvalue decreases and $\phi_{\mathrm{Pb}-\mathrm{vac}}(\varepsilon)$ is obtained as a function of the energy. The phase shift depends on the potential profile of the surface. Therefore, we check that it remains unaltered for thicknesses over approximately 2 ML. Finally, we determine, according to Eq. (B2), the shifts in the surface and interface positions, $\delta_{\mathrm{Pb}-\mathrm{vac}}(\epsilon)$ and $\delta_{\mathrm{Cu}(111)-\mathrm{Pb}}(\epsilon)$, respectively.

The identification of the quantum number $n$ used in Eq. (B1) is easy for states in free-standing Pb slabs. This is not the case for the QWSs in the $\mathrm{Pb} / \mathrm{Cu}(111)$ system because, in principle, it is not known how many states are hidden as resonances in the region of delocalized states in $\mathrm{Cu}$ [the white area between the gray ones in Fig. 2(b)]. Nevertheless, the local DOS integrated over the $\mathrm{Pb}$ overlayer reveals the number of the QWS resonances and, in addition, we have the possibility to plot the eigenfunctions in order to check the identification.

The dependences of the phase and surface position shifts on the energy eigenvalue are shown in Fig. 4. We note that nearly in the whole energy range the shift at the $\mathrm{Cu}(111)-\mathrm{Pb}$ interface is larger than that at the vacuum side. This means that the wave function penetration into $\mathrm{Cu}(111)$ is larger than the spill out into the vacuum. The shift of the effective $\mathrm{Pb}$ vacuum barrier is in agreement with the jellium-model calculations by Stratton, ${ }^{47}$ who obtained the average shift of $1.41 a_{0}$ between the bottom of the potential well (at $-9.5 \mathrm{eV}$ ) and the Fermi level. The present phase shift also agrees with the atomic pseudopotential results by Wei and Chou. ${ }^{23}$

The image-potential model [Eq. (B5)] reproduces our results quite well with the exception of the energies close to the vacuum level [Fig. 4(a)]. This is due to the exponential decay of the LDA potential into the vacuum. The empirical formula, Eq. (B6), gives good results at the $\mathrm{Cu}-\mathrm{Pb}$ interface if one corrects for the known downward shift of $\pi .{ }^{51}$ The finite-square-potential-well model does not produce phase shifts in agreement with the self-consistent results.

\section{B. Slab and infinite-square-potential well}

Combining the results of Fig. 4 and Eq. (B1) or (B2), the eigenvalues in Fig. 3 are reproduced. (The energy eigenvalues have to be shifted downward by the width $9.5 \mathrm{eV}$ of the occupied energy band). A good qualitative behavior is ob- 

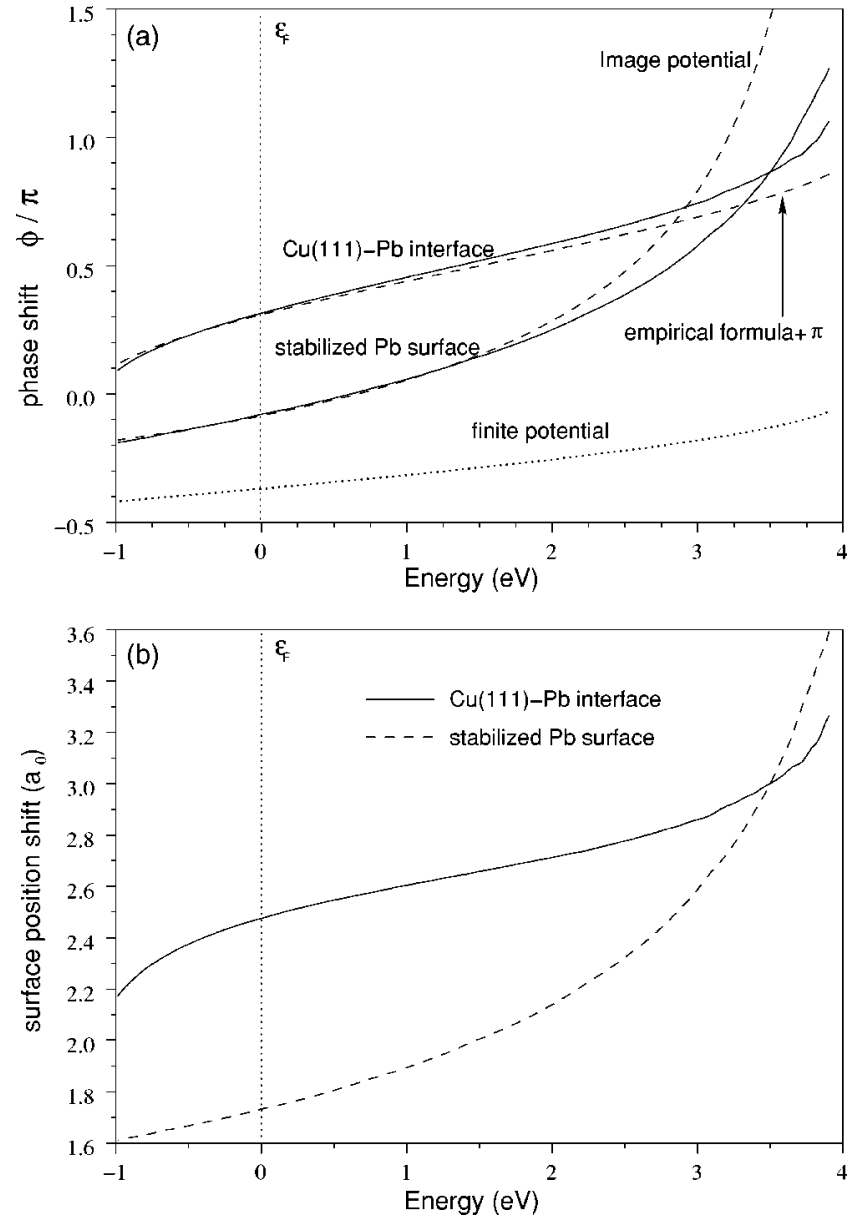

FIG. 4. (a) $\mathrm{Cu}(111)-\mathrm{Pb}$ and $\mathrm{Pb}$-vacuum phase shifts as a function of the QWS eigenenergy (with respect to the Fermi level). Results of our DFT calculations (solid line) and values obtained with Eqs. (B4) and (B5) for the Pb-vacuum interface and with Eq. (B6) for the $\mathrm{Cu}(111)-\mathrm{Pb}$ interface are shwon. (b) Shifts in the effective $\mathrm{Cu}(111)-\mathrm{Pb}$ interface and $\mathrm{Pb}$ surface positions are given by solid and dashed curves, respectively.

tained simply by using the mean effective width $D^{\prime}$, which is the ideal width $D$ (the width of the $\mathrm{Pb}$ positive background charge in the stabilized-jellium model) increased by $\sim 4.65 a_{0}$.

We compare in Fig. 5 our self-consistent DFT calculation for the $\mathrm{Pb} / \mathrm{Cu}(111)$ system with two simple model calculations. There are $5 \mathrm{ML}$ of $\mathrm{Pb}$ on the $\mathrm{Cu}(111)$ surface and we plot the electron density of the QWS at $\varepsilon=-0.53 \mathrm{eV}$. The corresponding state in a free-standing $\mathrm{Pb}$ slab is obtained by shifting the left $\mathrm{Pb}$-vacuum boundary by $0.75 a_{0}$ to the left in order to mimic the larger penetration of the wave functions into $\mathrm{Cu}(111)$ than into vacuum. The value of $0.75 a_{0}$ reproduces the correct infinite-barrier shift in Fig. 4(b). The eigenenergy of this $\mathrm{Pb}$ slab state is also $-0.53 \mathrm{eV}$. On the right surface, of course, both wave functions overlap and at the left surface the slab wave function tries to mimic, without oscillations, the decay of the wave function in the $\mathrm{Pb} / \mathrm{Cu}(111)$ system. The corresponding state calculated using the infinite-square-potential-well model with the appropriate shifts of the barriers $\left[1.66 a_{0}\right.$ and $2.37 a_{0}$ from Fig.

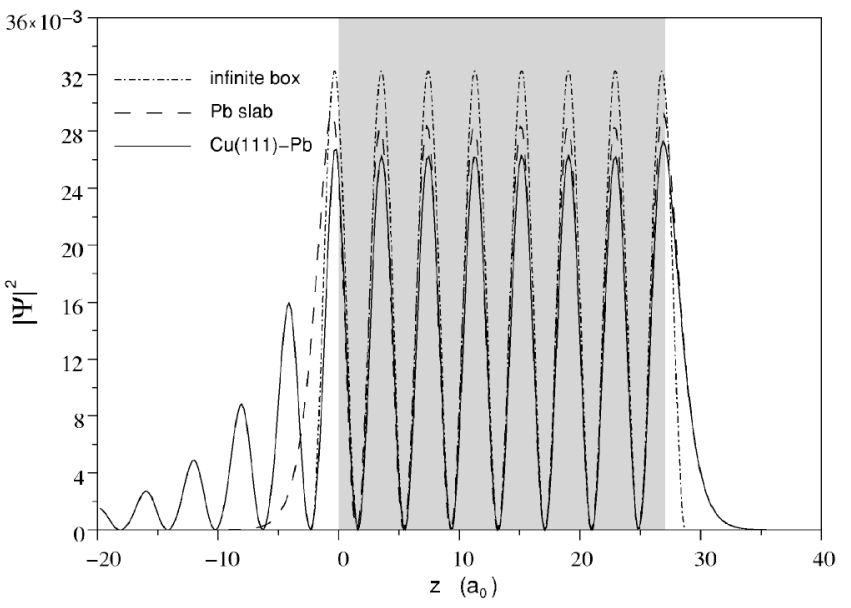

FIG. 5. Electron density of a QWS in the 5 ML thick Pb overlayer on $\mathrm{Cu}(111)$ as calculated self-consistently (solid line) and compared to the free-standing $\mathrm{Pb}$ slab (dashed line) and the infinitesquare-potential-well model (dashed-dotted line). The gray region denotes the $\mathrm{Pb}$ overlayer on $\mathrm{Cu}(111)$.

4(b)] is also given in Fig. 5. In conclusion, the two simple models with our parameters reproduce the eigenfunctions inside $\mathrm{Pb}$ and at the $\mathrm{Pb}$-vacuum barrier quite well, but not beyond the $\mathrm{Cu}(111)-\mathrm{Pb}$ interface. In order to describe properties related to the penetration of the wave functions into the substrate, the slab and infinite-square-potential-well models are inadequate.

\section{Importance of quantum numbers}

The model based on the phase shifts or on the effective width increase of the infinite-potential well reproduces the eigenvalues and eigenfunctions of the self-consistent calculation. Then, the good agreement of the self-consistently calculated eigenvalues with the experimental ones, ${ }^{20}$ allows one to fit the latter with the width increase $\delta_{0} \approx 4 a_{0}$. Nevertheless, Otero et al. ${ }^{20}$ obtain a good agreement with $\delta_{0}=0$.

To explain the contradiction we have compared the infinite-square-potential-well energy spectrum [Eq. (B2) obtained with $\left.\delta_{0}=0\right]$ to our model results corresponding to $\delta_{0}$ $\neq 0$. For example, in the latter the quantum number $n$ for the eigenstates with the nearly constant eigenenergy of $0.65 \mathrm{eV}$ are $n=4,7,10, \ldots$ for $2,4,6, \ldots$ ML of $\mathrm{Pb}$, respectively. Actually, this is in agreement with the pseudopotential calculations for free-standing Pb slabs. ${ }^{23}$ But $\delta_{0}=0$ gives the corresponding quantum numbers as $n=3,6,9, \ldots$, i.e., they are one unit smaller than the correct set. This explains why both models reproduce approximately the same experimental energy spectrum. The wavelength of the states $n=3,6,9, \ldots$ at $0.65 \mathrm{eV}$ in the infinite-potential well is $\lambda_{0.65}=7.2 a_{0}$. When we increase the width of the well by $\lambda_{0.65} / 2$, the states $n$ $=4,7,10, \ldots$ lie exactly at the same energy. This does not hold for energies far from $0.65 \mathrm{eV}$ (different wavelengths) and the eigenenergy spectra become different. Nevertheless, in the narrow energy window from -1 to $3 \mathrm{eV}$ scanned in experiments, the energy spectra of both models are very similar and fit the experimental results quite well. As a mat- 
ter of fact, an even better fit of experiments seems to be obtained by increasing the width of the potential well by $\lambda_{0.65}$ and using $n=5,8,11, \ldots$ states.

We want to stress the general conclusion that the energy spectra can be fitted with several sets of thickness and quantum numbers, but with the wrong set, other properties may be wrongly determined. In particular, the correct determination of the physical parameters reveals crucial in the description of the envelope function, which plays a key role in determining the energetics ${ }^{52}$ of the QWSs or the magnetic coupling in multilayer structures. ${ }^{53}$

\section{DETERMINATION OF CONFINEMENT BARRIERS}

\section{A. Calculation of effective width with $\delta_{0}$}

The disadvantage of the previous method for determining the positions of the confining barriers is a necessity to measure a large number of QWS eigenenergies in order to correctly label the quantum states. However, there exists methods in which the knowledge of the quantum number $n$ is not needed. ${ }^{11,21-23,54}$ We now consider this kind of method and offer an improved formula for their application.

From the energy difference $\Delta n$ between two consecutive states,

$$
\Delta_{n}=\varepsilon_{n+1}-\varepsilon_{n}=\frac{\pi^{2}(2 n+1)}{2\left(D+\delta_{0}\right)^{2}},
$$

an effective thickness of the infinite-potential well

$$
D^{\prime}=D+\delta_{0}=k_{0} \pi \Delta_{n}^{-1}
$$

is obtained, where $D$ is the ideal overlayer thickness or, in the case of experiments, the measured thickness and $k_{0}$ $=\pi(n+0.5) / D^{\prime}$ is a wave vector depending on $n$ and $D^{\prime} . \delta_{0}$ takes into account, e.g., the effect of the electron spill out into the vacuum or into the substrate, the unknown thickness of the wetting layer, and effects due to stress or relaxation at the boundaries.

We consider QWSs in overlayers of different thickness and within a given window, e.g., the QWSs shown in Fig. 3. Then, by plotting $D^{\prime}$ as a function of $D$ and by fitting to a straight line, the slope provides $k_{0}$ averaged over the energy window in question. For example, the theoretical data of Fig. 3 give $k_{0}=0.88 a_{0}^{-1}$, which corresponds to the kinetic energy of $10.5 \mathrm{eV}$, i.e., an energy $\sim 1 \mathrm{eV}$ above the Fermi level. The intersection of the dashed straight line with the vertical axis in Fig. 6 gives $\delta_{0}=14 a_{0}$. This value is much larger than the one obtained for the total shift of the potential barriers in Sec. IV. Namely, at the energy of $1 \mathrm{eV}$ above the Fermi level we obtain from Fig. 4(b) that $\delta_{0}=4.5 a_{0}$.

The method described above [Eq. (6)] has been used to determine the number of $\mathrm{Pb}$ wetting layers in STS experiments for $\mathrm{Pb} / \mathrm{Si}(111)$. Our finding is that the method overestimates the distance between the confinement barriers. This may explain the large values ${ }^{11,21-23}$ obtained by this scheme in comparison to other experiments. ${ }^{7-9}$ The reason for this disagreement is the basic fact that Eqs. (5) and (6) correspond to the infinite-potential well, whereas a finite potential well is closer to reality. The infinite-square-potential-well

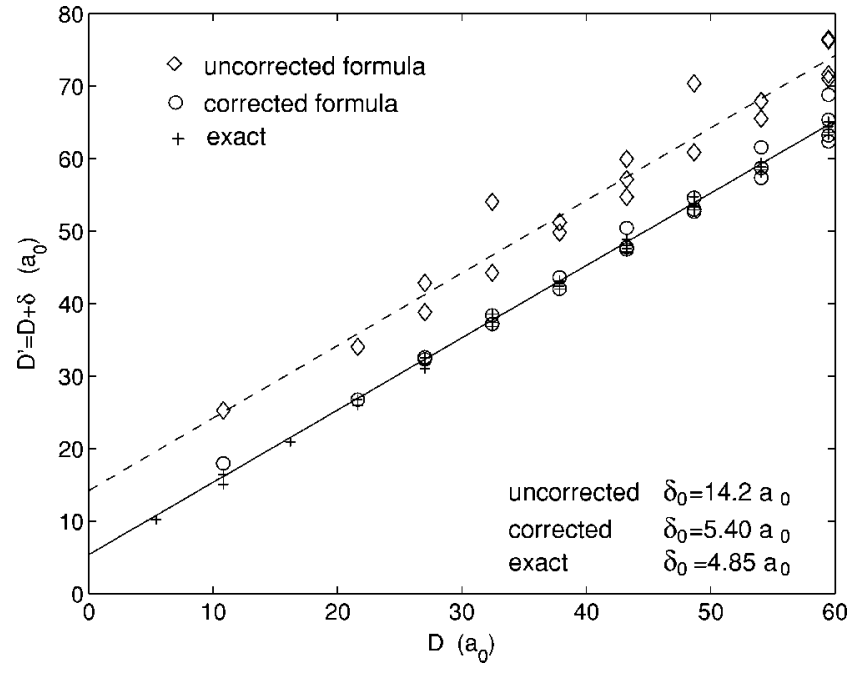

FIG. 6. Effective thickness calculated from the theoretical QWS energies in Fig. 3 (energy window from -1 to $3.5 \mathrm{eV}$ ) as a function of the $\mathrm{Pb}$ jellium slab thickness. Data and fits corresponding to Eq. (6) (diamonds, dashed line) and Eq. (8) (circles, solid line) are compared. The crosses are the exact effective thickness. Data up to $23 \mathrm{ML}$ are used in the fitting, but only data up to $11 \mathrm{ML}$ are plotted for clarity.

model with the shifts $\delta_{0}$ of the confining barriers reproduces quite accurately the energy spectrum as is demonstrated in Fig. 7. But the determination of the $\delta_{0}$ value from the real energy spectrum cannot be done using this scheme because the energy eigenvalue differences $\Delta_{n}$ between the consecutive states behave differently in the infinite-square-potential well and in real systems. The inset of Fig. 7 shows that the infinite-potential well results in a monotonically increasing $\Delta_{n}$, whereas in the more realistic jellium model $\Delta_{n}$ decreases close to the vacuum level. The wrong trend in the infinitesquare-potential-well model is compensated by the erroneously large effective width of the $\mathrm{Pb}$ slab or overlayer obtained by applying Eq. (6). In Secs. V B and V C we suggest a method to overcome this effect.

\section{B. Corrected formula for the calculation of $\delta_{0}$}

We propose to correct the overestimation inherent to Eq. (6) by introducing the effect of the finite potential barrier. This is done by assuming that the surface shift is energy dependent as $\delta=\delta_{0}+\delta(\varepsilon)$. Here, $\delta_{0}$ is the mean value we want to determine. Our aim is to obtain information about the electron-confinement strength through the $\delta_{0}$ parameter, which is energy-independent but reproduces satisfactorily the energy spectrum (see Fig. 6). Nevertheless, it is necessary to use the energy-dependent $\delta(\varepsilon)$ function to obtain relevant $\delta_{0}$ values.

The energy difference between the successive states can be obtained to the first order as

$$
\Delta_{n}=\frac{\mathrm{d} \varepsilon}{\mathrm{d} n}=\frac{\pi^{2} n}{D^{\prime 2}}-\frac{\pi^{2} n^{2}}{D^{\prime 3}} \dot{\delta}(\varepsilon) \frac{\mathrm{d} \varepsilon}{\mathrm{d} n},
$$

where $\dot{\delta}(\varepsilon)$ is the energy derivative of the surface shift. This equation has to be evaluated for a given $n$. Note that for the 


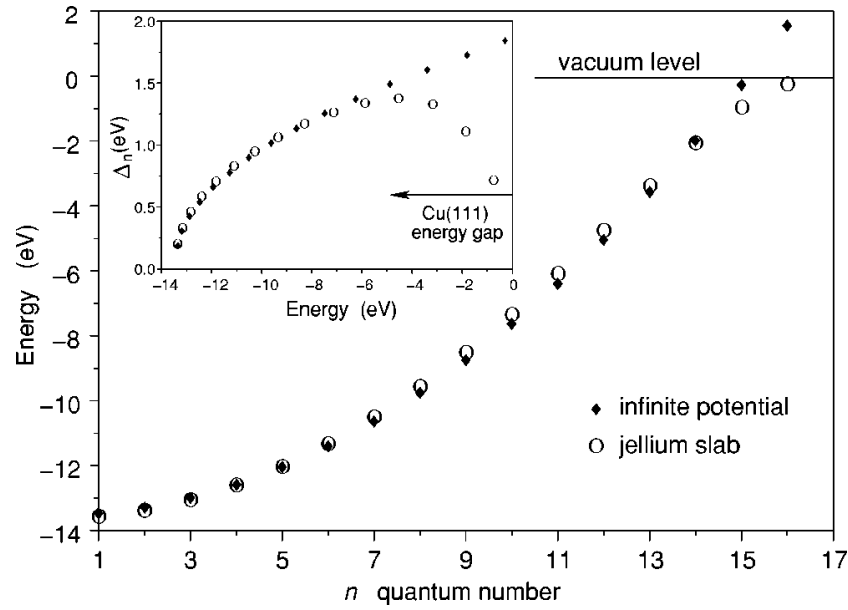

FIG. 7. Energy eigenvalues (bottoms of subbands) of a freestanding $8 \mathrm{ML}$ thick $\mathrm{Pb}$ slab as a function of the quantum number $n$. The open circles and the filled diamonds give the results of the stabilized-jellium and infinite-potential-well models, respectively. In the latter, the width of the well corresponds to $8 \mathrm{ML}$ widened by $4.5 a_{0}$ in order to take into account the electron spill out. The inset shows the energy differences between the consecutive states as a function of the energy. The energy range of the QWSs in the $\mathrm{Pb} / \mathrm{Cu}(111)$ system is given.

infinitely deep potential well $\dot{\delta}=0$, and we do not recover the exact result of Eq. (5). This deficiency is corrected by evaluating the right-hand side of Eq. (7) at $(n+1 / 2)$. The corresponding $\dot{\delta}$ is also evaluated at $\left(\varepsilon_{n+1}+\varepsilon_{n}\right) / 2$ using the selfconsistent $\delta$ values shown in Fig. 4(b). Then, rearranging the terms of Eq. (7) the corrected formula for the thickness casts as

$$
D^{\prime}=\pi k_{0} \Delta_{n}^{-1}-k_{0}^{2} \dot{\delta}=D+\delta .
$$

Here, it is accurate enough to use the $k_{0}$ obtained previously with Eq. (6). Omitting the dependence of $\delta$ on energy on the right-hand side of Eq. (8), i.e., setting $\delta=\delta_{0}$, we calculate the value of $\delta_{0}$ by fitting a straight line for $D^{\prime}$ as a function of $D$ (circles and the solid line in Fig. 6). The new mean value $\delta_{0}=5.4 a_{0}$ is consistent with the electron spill out calculated in Fig. 4(b) and it is much better than the value of $14 a_{0}$ obtained with the uncorrected Eq. (6). Namely, the exact values $D^{\prime}=\pi n / \sqrt{2 \varepsilon_{n}}$ (shown as crosses in Fig. 4) give $\delta_{0}$ $=4.85 a_{0}$.

\section{Analytical models for the $k_{0}^{2} \dot{\delta}$ correction}

In order to apply the corrected scheme of the previous subsection, the energy-dependent derivative $\dot{\delta}(\varepsilon)$ has to be known. We now study the reliability of different analytical models in its estimation, with the aim of extending the previous analysis to other substrates or overlayers without doing self-consistent electronic structure calculations.

The finite-square-potential-well model does not provide a correction large enough, because the potential is not a continuous function of energy. The $\dot{\delta}$ values are too small [see Fig. 4(a)]. The image-potential model [Eq. (B5)] and the

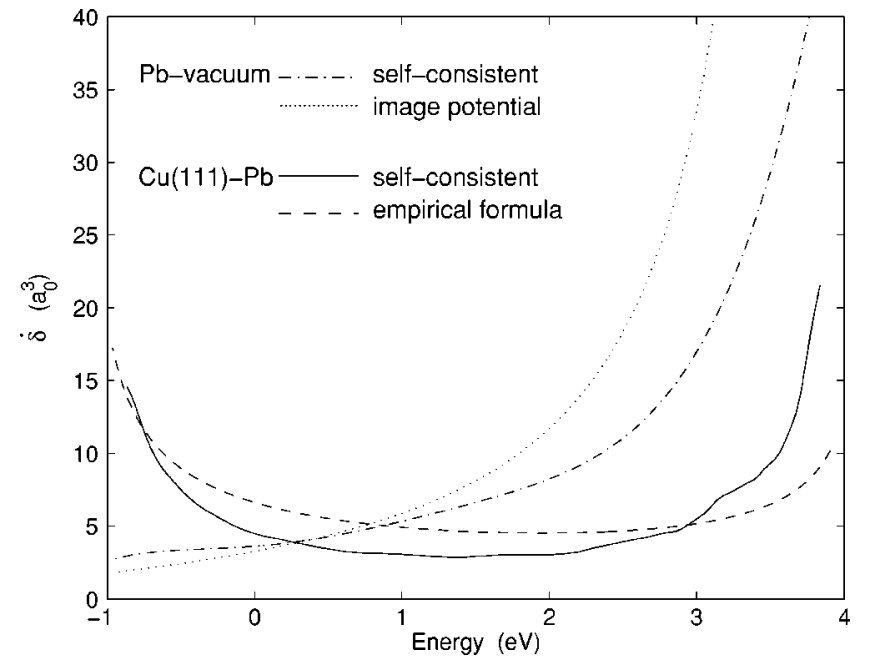

FIG. 8. Derivative $\dot{\delta}(\varepsilon)$ of the effective thickness with respect to the energy according to the self-consistent and to analytical models [Eqs. (B6) and (B5)] for the $\mathrm{Cu}(111)-\mathrm{Pb}$ interface and $\mathrm{Pb}$-vacuum surface. The energy origin is at the Fermi level.

empiric phase shifts [Eq. (B6)] provide results in a much better agreement with experiments. We want to emphazise that even if the analytical phase shifts may not produce the same $\delta_{0}$ values as the self-consistent calculations, they reproduce reasonably well the energy derivative $\dot{\delta}$ needed in the correction of Eq. (8).

Figure 8 shows the derivatives $\dot{\delta}$ from the self-consistent calculation and from the analytical models of Appendix B. The agreement is quite good, even if the derivatives of the self-consistent calculation are generally smaller. Comparing self-consistent and analytical curves we note that small differences in the phases [Fig. 4] produce big differences in Fig. 8. We recommend the use of QWSs at intermediate energies when determining the overlayer thickness because the correction is smaller in that region.

Table I shows a collection of $\delta_{0}$ values obtained for several systems using different approximations. In addition to our theoretical results for the $\mathrm{Pb} / \mathrm{Cu}(111)$ and the freestanding $\mathrm{Pb}$ slab systems we analyze our similar results for $\mathrm{Na} / \mathrm{Cu}(111)$ and the experimental QWS spectrum of $\mathrm{Pb} / \mathrm{Cu}(111)$ system. ${ }^{20}$ The "exact $\delta_{0}$ " values are obtained by fitting a straight line through the exact results $D^{\prime}=\pi n / \sqrt{2 \varepsilon_{n}}$. In general, the uncorrected Eq. (6) gives, for the $\mathrm{Pb}$ systems, $\delta_{0}$ values nearly three times larger than the exact one. In contrast, the corrected value offers a submonolayer accuracy. For the $\mathrm{Na} / \mathrm{Cu}(111)$ system the difference between the corrected and uncorrected $\delta_{0}$ is large as well. Although the wavelength of $\mathrm{Na}$ is larger than for $\mathrm{Pb}, \delta_{0}$ does not scale with it because the $\mathrm{Na} / \mathrm{Cu}(111)$ interface properties are different. Equation (8) applied to the experimental data gives $\delta_{0}$ $=7.3 a_{0}$ and $4.7 a_{0}$, with the numerical and analytical corrections, respectively. It is noticeable that the analytical result is closer to the exact value $\delta_{0}=4.0 a_{0}$. This can be explained by the correct description of the image potential by the analytical formula compared to the exponential LDA decay of the self-consistent one.

This method has been used mainly for $\mathrm{Pb} / \mathrm{Si}(111) .{ }^{11,21}$ For that system, values of 3 ML have been reported in STM 
TABLE I. $\delta_{0}$ values determining the effective widths of the infinite-potential wells fitting the QWS energy spectra, as obtained from different theoretical and experimental spectra by using the uncorrected and corrected schemes of Eqs. (6) and (8), respectively. The maximum overlayer thickness (in ML) used in the fitting are given in the second column. The numbers in parenthesis give the linear regression errors. The QWS of the DFT calculations used in the fitting span the energy range from $\sim 1 \mathrm{eV}$ below Fermi level, i.e., from the bottom of the $\mathrm{Cu}(111)$ band gap, to $\sim 1 \mathrm{eV}$ below vacuum level (for $\mathrm{Pb} / \mathrm{Cu}(111)$ corresponds to the experimently measured energy range of QWSs).

\begin{tabular}{|c|c|c|c|c|c|}
\hline System & Up to & $\begin{array}{c}\delta_{0} \text { from Eq. (6) } \\
\text { uncorrected }\end{array}$ & $\begin{array}{c}\delta_{0} \text { from Eq. }(8) \\
\text { numerical correction }\end{array}$ & $\begin{array}{c}\delta_{0} \text { from Eq. (8) } \\
\text { analytical correction }\end{array}$ & Exact $\delta_{0}$ \\
\hline Free-standing $\mathrm{Pb}$ & $10 \mathrm{ML}$ & $12(3)$ & $5.5(0.6)$ & $6(3)$ & $3.9(0.3)$ \\
\hline $\mathrm{Pb} / \mathrm{Cu}(111)$ & $23 \mathrm{ML}$ & $12(1)$ & $5(2)$ & $3(2)$ & $4.7(0.1)$ \\
\hline $\mathrm{Pb} / \mathrm{Cu}(111)$ & $10 \mathrm{ML}$ & $13(1)$ & $6(1)$ & $4(2)$ & $4.8(0.3)$ \\
\hline Experiments $^{20}$ & $24 \mathrm{ML}$ & $15(5)$ & $7(5)$ & $5(5)$ & $4.0(0.2)$ \\
\hline $\mathrm{Na} / \mathrm{Cu}(111)$ & $20 \mathrm{ML}$ & $7(3)$ & $1(3)$ & $3(4)$ & $1.2(0.3)$ \\
\hline
\end{tabular}

experiments, but with the corrected scheme we propose, it would be of one ML, as reported in other experiments. ${ }^{7-9}$ In a recent paper $^{54}$ concerning $\mathrm{Pb} / \mathrm{Cu}(111)$, the energy differences are used to estimate the thickness of the slab to be 22 ML, while the comparison to our calculation suggests that it is only 20 ML.

When analyzing experiments, the $\delta_{0}$ value obtained by the corrected scheme of Eq. (8) can be used as an initial parameter to determine the $n$ quantum number. Then, employing the exact equation to fit the energy spectrum, improved results are obtained.

\section{CONCLUSIONS}

We have performed self-consistent DFT calculations to study the confinement barriers of electrons in $\mathrm{Pb}$ islands grown on the $\mathrm{Cu}(111)$ substrate. Additional calculations have been done for free-standing $\mathrm{Pb}$ slabs, for comparison. $\mathrm{Pb}$ has been described by stabilized jellium and the $\mathrm{Cu}(111)$ substrate by a 1D pseudopotential. The model reproduces the most important physical properties and gives results in good agreement with experiments.

The energies and wave functions of the quantum well states in the $\mathrm{Pb}$ slabs characterize the confinement barriers at the $\mathrm{Pb}$-vacuum surface and at the $\mathrm{Cu}(111)-\mathrm{Pb}$ interface. We have analyzed these states by using the phase accumulation model and by determining effective widths of infinitepotential wells reproducing the energies. The $\mathrm{Pb}$-vacuum phase shift is in good agreement with more realistic pseudopotential calculations. The $\mathrm{Cu}(111)-\mathrm{Pb}$ phase shift or the effective width of the potential well accounts for the confining strength of the $\mathrm{Cu}(111)$ energy gap. This strength is weaker than that of the $\mathrm{Pb}$-vacuum barrier.

The information provided by our calculations and analysis allows one to improve the interpretation of QWS spectra measured by scanning tunneling spectroscopy. More specifically, we have shown that the formula commonly used in the literature results in the overestimation of the effective width of the infinite-potential well. We have offered an alternate expression to correct that deficiency, especially important for high electronic density (small $\lambda_{F}$ ) metals. The results obtained can be used to estimate the width of the potential well and to determine the quantum numbers for a more accurate analysis of the confinement barriers.

Finally, the 1D-pseudopotential scheme provides a method for future studies of nanostructures on solid surfaces. This work is focused on the $\mathrm{Pb} / \mathrm{Cu}(111)$ system, but the analytical expressions derived from our analysis are general and the results can be qualitatively applied to metallic nanoislands on solid substrates, probed for instance with photoemission or STS.

\section{ACKNOWLEDGMENTS}

The authors are grateful to R. Miranda for his valuable comments. We acknowledge partial support by the University of the Basque Country (9/UPV00224.310-14553/2002), the Basque Hezkuntza Unibertsitate eta Ikerkuntza Saila and the Spanish Ministerio de Ciencia y Tecnología (MAT 20010946 and MAT2002-04087-CO2-O1). This work was also partially supported by the Academy of Finland through its Centre of Excellence Program (2000-2005) and by the European Community sixth Framework Network of Excellence NANOQUANTA (NMP4-CT-2004-500198).

\section{APPENDIX A: GENERATION OF THE CU(111) 1D PSEUDOPOTENTIAL}

We have to obtain, first, an unscreened pseudopotential for periodic bulk calculations. Chulkov et al. ${ }^{55,56}$ proposed a fully screened $1 \mathrm{D}$-model potential that varies only in the $z$ direction perpendicular to the surface. The model potential is successfully used to study, for example, the dielectric response function and lifetimes of excited electron states. ${ }^{55-58}$ The crucial point here is the proper description of the energy band gap and work function. Moreover, the wave functions are correctly described not only outside the substrate, but also inside it. This is an important ingredient in the present application. Here we skip the surface part of the 1D pseudopotential because we cover the $\mathrm{Cu}(111)$ surface with several ML of Pb. Nevertheless, it is also possible to build a pseudopotential that reproduces the surface and image states. ${ }^{59}$ The bulk oscillating function of the $1 \mathrm{D}$-model potential ${ }^{55,56}$ is 


$$
V_{\text {model }}(z)=A_{10}+A_{1} \cos \left(\frac{2 \pi}{d} z\right), \quad \frac{-d}{2}<z<\frac{d}{2},
$$

where $d=3.943 a_{0}$ is the interlayer spacing in $\mathrm{Cu}$ in the [111] direction and $A_{10}$ and $A_{1}$ are fitting parameters. Using periodic boundary conditions at $\pm d / 2$ the Kohn-Sham equations are solved for the fixed $V_{\text {eff }}(z)=V_{\text {model }}(z)$ potential. With the eigenfunctions obtained and with the experimental work function we calculate the electron-density profile. Integrating over $z$ we obtain the mean density with $r_{s}=2.55 a_{0}$. This value is close to the experimental $r_{s}=2.67 a_{0}$ for $\mathrm{Cu} 4 \mathrm{~s}$ electrons.

Once we have computed the density, it is straightforward to obtain the corresponding $V_{x c}(z)$ potential. It is more challenging to calculate the Hartree $V_{H}(z)$ term because in the absence of vacuum the height of the surface dipole-barrier and the energy origin for the Hartree term inside the bulk are not known. To solve the problem we fix, provisionally, the zero of the Hartree potential to occur at the boundaries of the periodic cell. After adding a homogeneous neutralizing positive background of $r_{s}=2.55 a_{0}$, the Hartree potential can be evaluated. Finally, we can calculate from Eq. (3) the unscreened and periodic pseudopotential $V_{p s}(z)$ by subtracting from the effective potential the $V_{H}(z)$ and $V_{x c}(z)$ terms.

The pseudopotential obtained cannot be used in slab calculations yet because the zero of the $V_{H}(z)$ potential was arbitrarily chosen in the previous step.

In a second step we build a semi-infinite $\mathrm{Cu}(111)$ slab by repeating the pseudopotential of $1 \mathrm{ML}$ of $\mathrm{Cu}$. The slab is thick enough to avoid interaction between surfaces and finite-size effects in determining the band structure. Then, enough vacuum is added on both sides to annul boundary effects at the borders of the calculation volume. In the selfconsistent calculation for this system the electron density spills out of $\mathrm{Cu}(111)$ to the vacuum, giving rise to the dipole Coulomb barrier, which shifts the Fermi level (and the whole band structure including the energy gap) to the position that is not yet correct with respect to the vacuum level.

To correct the work function we shift the pseudopotential by a constant inside the $\mathrm{Cu}$ slab. Here we define the $\mathrm{Cu}(111)$ edge to be at half an interlayer spacing beyond the last atom plane. But the pseudopotential shift also changes the electron spilling into the vacuum and the dipole barrier. Thus, we find the potential shift iteratively so that the experimental value for the work function is recovered.

\section{APPENDIX B: PHASE ACCUMULATION MODEL FOR CONFINEMENT BARRIERS}

The phase accumulation model ${ }^{38}$ includes the features of the QWSs in a simple way, through the phase shifts at the limiting surfaces. For a $\mathrm{Pb}$ slab over $\mathrm{Cu}(111)$ it yields the following expression:

$$
2 k_{z} D+\phi_{\mathrm{Cu}(111)-\mathrm{Pb}}+\phi_{\mathrm{Pb}-\mathrm{vac}}=2 \pi(n-1),
$$

where $k_{z}$ is the wave vector corresponding to the QWS kinetic energy, $D$ is the width of the potential well to model the $\mathrm{Pb}$ film, $\phi_{\mathrm{Pb} \text {-vac }}$ and $\phi_{\mathrm{Cu}(111)-\mathrm{Pb}}$ are the phases of the eigenfunction accumulated at the $\mathrm{Pb}$ overlayer-vacuum and the $\mathrm{Cu}$-substrate- $\mathrm{Pb}$-overlayer interfaces, respectively, and $n$ $=1,2,3, \ldots$ is the quantum number of the QWS. For an infinitely deep square-potential well, the phase accumulated on each surface is $-\pi$. It is more intuitive to apply the infinitely deep square-potential well so that $\phi_{\mathrm{Pb}-\mathrm{vac}}=\phi_{\mathrm{Cu}-\mathrm{Pb}}=-\pi$, but using an effective width $D^{\prime}=D+\delta_{\mathrm{Cu}(111)-\mathrm{Pb}}+\delta_{\mathrm{Pb}-\mathrm{vac}}$. Then Eq. (B1) gives

$$
\delta_{\mathrm{Cu}(111)-\mathrm{Pb}}+\delta_{\mathrm{Pb}-\mathrm{vac}}=\frac{\pi n}{k_{z}}-D .
$$

Here, $\delta_{\mathrm{Cu}(111)-\mathrm{Pb}}$ and $\delta_{\mathrm{Pb}-\mathrm{vac}}$ arise from the wave function penetration into the $\mathrm{Cu}(111)$ substrate and the spill out into the vacuum, respectively. The idea is that $D^{\prime}$ should give a reliable estimate of the actual thickness of the overlayer. Thus, as the phase shifts, the effective well width also depends on the QWS eigenenergy $\varepsilon_{n}$. It has been shown that the energy spectrum is very sensitive to the positions of the barriers and relatively insensitive to the barrier height. ${ }^{20}$ Therefore, a mean surface shift $\delta_{0}$ can accurately reproduce the spectrum. According to Eqs. (B1) and (B2) the surface position shifts and phase shifts are related by

$$
\delta=\frac{\phi+\pi}{2 k_{z}}
$$

Below we give two analytical expressions for the surfacevacuum phase shift, derived within the finite-potential-step and the image-potential models. The former gives the energy-dependent phase shift ${ }^{20,60}$

$$
\phi_{\mathrm{Pb}-\mathrm{vac}}=2 \arctan \left(-\frac{k_{\mathrm{vac}}}{k_{z}}\right),
$$

where $k_{\mathrm{vac}}=\sqrt{2|\varepsilon|}$ and the energy eigenvalue $\varepsilon$ is measured with respect to the vacuum level. In Ref. 20 this model has also been used for the analysis of the $\mathrm{Cu}-\mathrm{Pb}$ interface barrier. The image-potential model gives the phase shift ${ }^{61}$

$$
\phi_{\mathrm{Pb}-\mathrm{vac}}=\pi \sqrt{\frac{3.4 \mathrm{eV}}{|\varepsilon|}}-\pi \text {. }
$$

The phase shift corresponding to the QWS's wave functions penetrating into the $\mathrm{Cu}(111)$ depends on the position of the QWS energy eigenvalue relative to the energy band gap. For example, the empiric formula ${ }^{61}$

$$
\phi_{\mathrm{Cu}(111)-\mathrm{Pb}}=2 \arcsin \sqrt{\frac{\varepsilon-\varepsilon_{L}}{\varepsilon_{U}-\varepsilon_{L}}}-\pi
$$

has been used, where $\varepsilon_{U}$ and $\varepsilon_{L}$ are the upper and lower edges of the band gap, respectively. 
*Electronic address: eoa@we.lc.ehu.es

${ }^{1}$ T. C. Chiang, Surf. Sci. Rep. 39, 181 (2000).

${ }^{2}$ F. J. Himpsel, J. E. Ortega, G. J. Mankey, and R. F. Willis, Adv. Phys. 47, 511 (1998).

${ }^{3}$ R. Otero, A. L. Vázquez de Parga, and R. Miranda, Phys. Rev. B 66, 115401 (2002).

${ }^{4}$ B. J. Hinch, C. Koziol, J. P. Toennies, and G. Zhang, Europhys. Lett. 10, 341 (1989).

${ }^{5}$ K. Budde, E. Abram, V. Yeh, and M. C. Tringides, Phys. Rev. B 61, R10 602 (2000).

${ }^{6}$ D. A. Luh, T. Miller, J. J. Paggel, M. Y. Chou, and T. C. Chiang, Science 292, 1131 (2001).

${ }^{7}$ V. Yeh, M. Hupalo, E. H. Conrad, and M. C. Tringides, Surf. Sci. 551, 23 (2004).

${ }^{8}$ M. Hupalo and M. C. Tringides, Phys. Rev. B 65, 115406 (2002).

${ }^{9}$ A. Mans, J. H. Dil, A. R. H. F. Ettema, and H. H. Weitering, Phys. Rev. B 66, 195410 (2002).

${ }^{10}$ M. Hupalo, S. Kremmer, V. Yeh, L. Berbil-Bautista, E. Abram, and M. C. Tringides, Surf. Sci. 493, 526 (2001).

${ }^{11}$ W. B. Su, S. H. Chang, W. B. Jian, C. S. Chang, L. J. Chen, and T. T. Tsong, Phys. Rev. Lett. 86, 5116 (2001).

${ }^{12}$ A. Menzel, M. Kammler, E. H. Conrad, V. Yeh, M. Hupalo, and M. C. Tringides, Phys. Rev. B 67, 165314 (2003).

${ }^{13}$ E. Ogando, N. Zabala, E. V. Chulkov, and M. J. Puska, Phys. Rev. B 69, 153410 (2004).

${ }^{14}$ L. Gavioli, K. R. Kimberlin, M. C. Tringides, J. F. Wendelken, and Z. Zhang, Phys. Rev. Lett. 82, 129 (1999).

${ }^{15}$ I. Matsuda, H. W. Yeom, T. Tanikawa, K. Tono, T. Nagao, S. Hasegawa, and T. Ohta, Phys. Rev. B 63, 125325 (2004).

${ }^{16}$ T. Nagao, J. T. Sadowski, M. Saito, S. Yaginuma, Y. Fujikawa, T. Kogure, T. Ohno, S. Hasegawa, and T. Sakurai, Phys. Rev. Lett. 93, 105501 (2004).

${ }^{17}$ P. Czoschke, H. Hong, L. Basile, and T. C. Chiang, Phys. Rev. Lett. 93, 036103 (2004).

${ }^{18}$ P. Czoschke, H. Hong, L. Basile, and T. C. Chiang, Phys. Rev. Lett. 91, 226801 (2003).

${ }^{19}$ L. Floreano, D. Cvetko, F. Bruno, G. Bavdek, A. Cossaro, R. Gotter, A. Verdini, and A. Morgante, Prog. Surf. Sci. 72, 135 (2004).

${ }^{20}$ R. Otero, A. L. Vázquez de Parga, and R. Miranda, Surf. Sci. 447, 143 (2000).

${ }^{21}$ I. B. Altfeder, K. A. Matveev, and D. M. Chen, Phys. Rev. Lett. 78, 2815 (1997).

${ }^{22}$ W. B. Su, S. H. Chang, C. S. Chang, L. J. Chen, and T. T. Tsong, Jpn. J. Appl. Phys., Part 1 40, 4299 (2001).

${ }^{23}$ C. M. Wei and M. Y. Chou, Phys. Rev. B 66, 233408 (2002).

${ }^{24}$ G. Materzanini, P. Saalfrank, and P. J. D. Lindan, Phys. Rev. B 63, 235405 (2001).

${ }^{25}$ P. J. Feibelman, Phys. Rev. B 27, 1991 (1983).

${ }^{26}$ P. J. Feibelman and D. R. Hamann, Phys. Rev. B 29, 6463 (1984).

${ }^{27}$ A. Kiejna, J. Peisert, and P. Scharoch, Surf. Sci. 432, 54 (1999).

${ }^{28}$ J. C. Boettger and S. B. Trickey, Phys. Rev. B 45, 1363 (1992).

${ }^{29}$ J. C. Boettger, Phys. Rev. B 53, 13133 (1996).
${ }^{30}$ P. Saalfrank, Surf. Sci. 274, 449 (1992).

${ }^{31}$ I. P. Batra, S. Ciraci, G. P. Srivastava, J. S. Nelson, and C. Y. Fong, Phys. Rev. B 34, 8246 (1986).

${ }^{32}$ F. K. Schulte, Surf. Sci. 55, 427 (1976).

${ }^{33}$ I. Sarría, C. Henriques, C. Fiolhais, and J. M. Pitarke, Phys. Rev. B 62, 1699 (2000).

${ }^{34}$ C. Fiolhais, C. Henriques, I. Sarría, and J. M. Pitarke, Prog. Surf. Sci. 67, 285 (2001).

${ }^{35}$ K. F. Wojciechowski and H. Bogdanów, Surf. Sci. 397, 53 (1998).

${ }^{36}$ S. Ciraci and I. P. Batra, Phys. Rev. B 33, 4294 (1986).

${ }^{37}$ H. Hong, C. M. Wei, M. Y. Chou, Z. Wu, L. Basile, H. Chen, M. Holt, and T. C. Chiang, Phys. Rev. Lett. 90, 076104 (2003).

${ }^{38}$ P. M. Echenique and J. B. Pendry, J. Phys. C 11, 2065 (1978).

${ }^{39}$ R. O. Jones and O. Gunnarsson, Rev. Mod. Phys. 61, 689 (1989).

${ }^{40}$ Y. Wang and J. P. Perdew, Phys. Rev. B 43, 8911 (1991).

${ }^{41}$ D. M. Ceperley and B. J. Alder, Phys. Rev. Lett. 45, 566 (1980).

${ }^{42}$ J. Mandel and S. F. McCormick, J. Comput. Phys. 80, 442 (1989).

${ }^{43}$ M. Heiskanen, T. Torsti, M. J. Puska, and R. M. Nieminen, Phys. Rev. B 63, 245106 (2001).

${ }^{44}$ T. Torsti, M. Heiskanen, M. J. Puska, and R. M. Nieminen, Int. J. Quantum Chem. 91, 171 (2003).

${ }^{45}$ J. P. Perdew, H. Q. Tran, and E. D. Smith, Phys. Rev. B 42, 11627 (1990).

${ }^{46}$ H. B. Shore and J. H. Rose, Phys. Rev. Lett. 66, 2519 (1991).

${ }^{47}$ R. Stratton, Phys. Rev. Lett. 19, 556 (1965).

${ }^{48}$ E. Ogando, N. Zabala, and M. J. Puska, Nanotechnology 13, 363 (2002).

${ }^{49}$ A. García-Martín, J. A. Torres, and J. J. Sáenz, Phys. Rev. B 54, 13448 (1996).

${ }^{50}$ M. Brack, Rev. Mod. Phys. 65, 677 (1993).

${ }^{51}$ J. B. Pendry and S. J. Gurman, Surf. Sci. 49, 87 (1975).

${ }^{52}$ R. K. Kawakami, E. Rotenberg, H. J. Choi, E. J. EscorciaAparicio, M. O. Bowen, J. H. Wolfe, E. Arenholz, Z. D. Zhang, N. V. Smith, and Z. Q. Qiu, Nature (London) 398, 132 (1999).

${ }^{53}$ J. E. Ortega, F. J. Himpsel, G. J. Mankey, and R. F. Willis, Phys. Rev. B 47, 1540 (1993).

${ }^{54}$ J. H. Dil, J. W. Kim, S. Gohkale, M. Tallarida, and K. Horn, Phys. Rev. B 70, 045405 (2004).

${ }^{55}$ E. V. Chulkov, V. M. Silkin, and P. M. Echenique, Surf. Sci. 437, 330 (1999)

${ }^{56}$ E. V. Chulkov, V. M. Silkin, and P. M. Echenique, Surf. Sci. 391, L1217 (1997).

${ }^{57}$ E. V. Chulkov, V. M. Silkin, and M. Machado, Surf. Sci. 482485, 693 (2001).

${ }^{58}$ E. V. Chulkov, I. Sarría, V. M. Silkin, J. M. Pitarke, and P. M. Echenique, Phys. Rev. Lett. 80, 4947 (1998).

${ }^{59}$ E. Ogando, N. Zabala, E. V. Chulkov, and M. J. Puska (unpublished).

${ }^{60}$ N. V. Smith, Phys. Rev. B 32, 3549 (1985).

${ }^{61}$ P. M. Echenique and J. B. Pendry, Prog. Surf. Sci. 32, 111 (1989). 